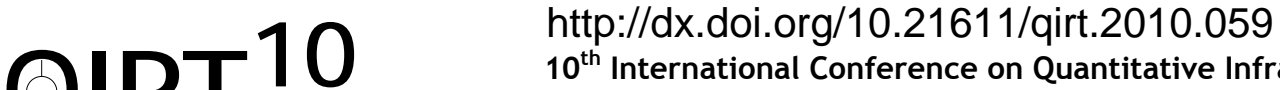 \\ $10^{\text {th }}$ International Conference on Quantitative InfraRed Thermography \\ July 27-30, 2010, Québec (Canada)
}

\section{IR wall heat transfer in swirling impinging jets}

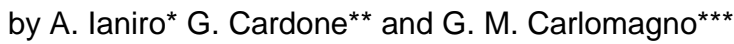

\begin{abstract}
*DIAS, University of Naples "Federico II", Via Claudio 21, 80125,Naples, Italy, andrea.ianiro@unina.it **DIAS, University of Naples "Federico II", Piazzale Tecchio 80, 80125, Naples, Italy, gcardone@unina.it

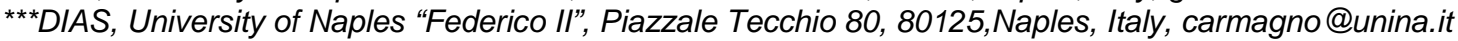

\begin{abstract}
The purpose of this work is the experimental analysis of the heat transfer between a flat plate and a swirling air jet impinging on it. A swirling nozzle based on helical inserts, of easy application in industrial environment, has been developed and experimental measurements are done at a fixed value of Reynolds number $(R e$ is based on the flow rate and on the nozzle diameter, $R e=30000)$ for five values of Swirl number $S(0,0.2,0.4,0.6$ and 0.8$)$ and five values of nozzle to plate distance (2, 4, 6, 8 and 10 diameters). Experimental measurements are done by means of IR thermography with the heated thin foil sensor. The different behaviors of multi-channel jets, weak swirl jets and strong swirl jets are shown. The uniformity of the heat transfer and the global heat transfer rate are quantified as mean value and standard deviation percentage of Nusselt number on the impinged area. The performances in term of heat transfer uniformity and heat transfer rate provided by swirling jets are also presented and analyzed. The dependence of the heat transfer rate and uniformity on the swirl number is also explained.
\end{abstract}

\section{Introduction}

The high heat transfer rate that is possible to obtain with impinging jets is well recognized and explained in a wide scientific literature [1]. It makes the use of jets very diffused in several industrial applications such as drying, tempering and turbine blades cooling. About single, rows and multiple jets a huge quantity of data and correlations for heat and mass transfer is available [2,3].

The flow structure of impinging jets is characterized by the nozzle diameter and the nozzle to plate distance. The structures of impinging axis-symmetric jets can be subdivided in three characteristic regions: the free jet region, the stagnation (impingement) region and the wall jet region (see Fig. 1). The stagnation region and the wall jet region are characterized by very different heat transfer rates so one of the main limitations of jet impingement heat transfer is the radial non-uniformity.

For some applications such as electronic cooling and chemical vapor deposition high values and radial uniformity of heat and mass transfer are required. In order to achieve an higher heat transfer radial uniformity the swirling impinging jet could be a possible solution. Swirling impinging jets are characterized by tangential velocity components that cause a spiralshaped motion and the broadening of the impingement region and of the wall jet region (Fig. 1); this aspect is coupled, especially near the stagnation point, with an axial flux weakening as well.

Generally it is possible to obtain a swirl flow by mixing two air flows or by using an insert inside nozzle able to produce the swirling motion. A swirling impinging jet has been designed for the first time by Ward and Mahmood [4], based on the concept of mixing the two air flows; the measured radial distribution of local Nusselt number was just slightly more uniform and its values were significantly lower. An improved swirling nozzle, based on a cylindrical plug with four narrow channels, was developed by Huang and El Genk [5] and the experimental measurements demonstrated the improvement of the radial uniformity and also the increase of the surface average Nusselt number. Also Lee et al. [6] studied the heat transfer distribution related to swirling impinging jets based on the concept of an insert inside the jet nozzle; the heat transfer uniformity given by the swirl is assessed and it is demonstrated that the heat transfer distribution is strictly depending on the Swirl number $S$ which is a nondimensional number representing axial flux of swirl momentum divided by axial flux of axial momentum, times the equivalent nozzle radius [7]. Wen and Jang [8] designed swirling strips to be insert in the jet nozzle and showed that the solution of the crossed swirling strips has better heat transfer performances respect to standard jets. In literature of swirling impinging jets heat transfer data are reported only in terms of radial distribution even if the behavior of the flow field should require two dimensional measurements.

In this work some helical inserts based on the concept of the crossed swirling strips are designed and developed by means of quick prototyping. Experimental two dimensional measurements of convective heat transfer between a flat plate and a swirling air jet impinging on it are carried out for different nozzle to plate distances and different Swirl numbers. Experimental measurements are done by means of IR thermography and with the "heated thin foil" heat transfer sensor [9]. Measured data allowed to analyze the influence of the Swirl number and of the nozzle to plate distance on the heat transfer 
distribution and on the flow topological structure. Data are reported as Nusselt number surface maps, surface averaged Nusselt number and surface standard deviation of Nusselt number.

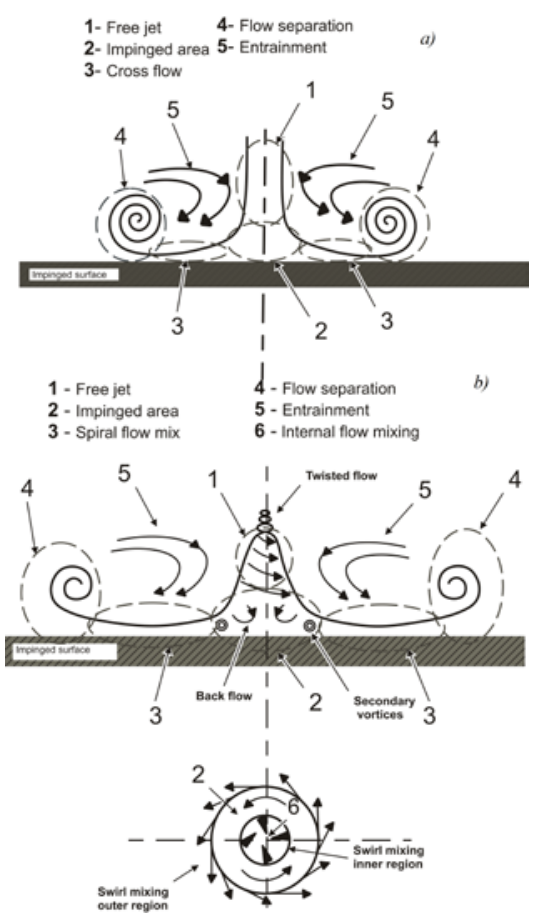

Fig. 1. Flow topological structures: a) conventional impinging jets b)swirling impinging jets

\section{Experimental apparatus and measurement technique}

The experimental apparatus, sketched in Fig. 2, includes a thin constantan foil $(200 \mathrm{~mm}$ wide, $470 \mathrm{~mm}$ long and $0.050 \mathrm{~mm}$ thick). This foil, which constitutes the target plate, is heated by passing an electric current through it and cooled by the air jet which is impinging on it. The plate is horizontally positioned with the impinging jet placed vertically below so as to minimize the recirculation of hot spent air. A suitable stiffening tool is employed to assure the foil surface flatness. The cooling air, supplied by a compressor, goes through a pressure regulating valve, a heat exchanger that keeps the (total) temperature of the jet close to that of the ambient room air, then to a plenum chamber where pressure and temperature are metered and finally impinges on the target plate. The swirling flow is obtained by passing the air in a cylindrical nozzle (exit diameter $D=18 \mathrm{~mm}$ ) with inside a helical insert (swirl generator) based on the concept of the crossed swirling strips. Five dimensionless nozzle-to-plate distances $z=Z / D$ (where $Z$ is distance between nozzle exit and the target plate) are considered, namely $2,4,6,8$ and 10 . The mass flow rate $\dot{m}$ is maintained constant so as to have always the same value of the Reynolds number, defined as $R e=4 \dot{m} /(\pi \mu D)$ (where $\mu$ is the viscosity coefficient of air), equal to 30000 . Using five different swirl generators, tests were performed for 5 different values of Swirl number $S$, namely 0, 0.2, 0.4, 0.6, 0.8; in fact, according to the formula proposed by Gupta [7], swirl number can be expressed as:

$$
S=\frac{2}{3}\left[\frac{1-(d / D)^{3}}{1-(d / D)^{2}}\right] \tan \theta
$$

where $d$ and $D$ are nozzle and vane pack hub diameters respectively and $\theta$ is the swirling angle as shown in Fig. 3 . 


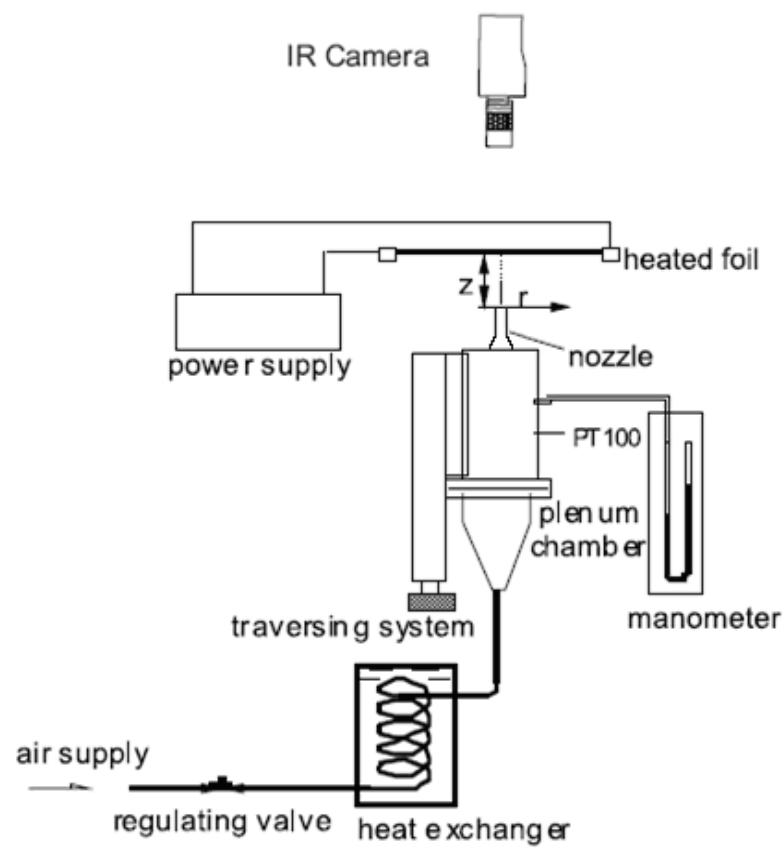

Fig. 2. Test set-up for heat transfer measurements
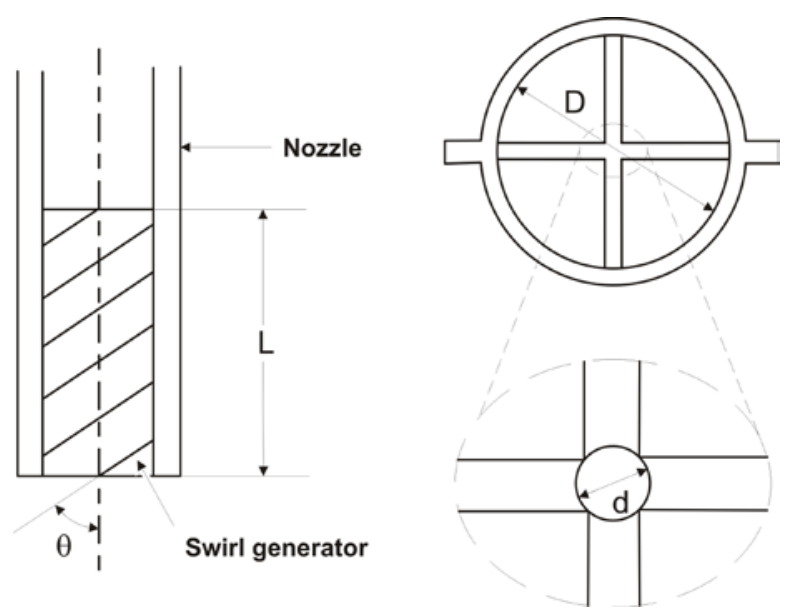

Fig. 3. Swirling nozzle........

A thermographic system (IR camera), based on a FLIR SC6000 LW (FPA of 640x512 pixels, working the 8-12 $\mu \mathrm{m}$ band) is employed to measure the foil surface temperature. In the present case IR camera is used in conjunction to the heated thin foil sensor [9], which is able to measure the average (in time) local convective heat transfer coefficient $h$ between a thin metallic foil, heated by Joule effect, and the air jet impinging on it. In fact by an energy balance referring at Fig. 4 it comes the formula:

$$
h=\frac{\dot{q}-\dot{q}_{l}}{T_{w}-T_{a w}}
$$

with $\dot{q}$ the Joule heating and $\dot{q}_{l}$ the losses which are due to radiation $\dot{q}_{r}$, natural convection on the rear foil surface $\dot{q}_{n}$ and tangential conduction $\dot{q}_{k}, T_{w}$ the wall temperature and $T_{a w}$ the adiabatic wall temperature. In the present case, $\dot{q}_{r}$ is about $5 \%, \dot{q}_{k}$ is about $1.5 \%$ and $\dot{q}_{n}$ is about $3 \%$ of $\dot{q}$.

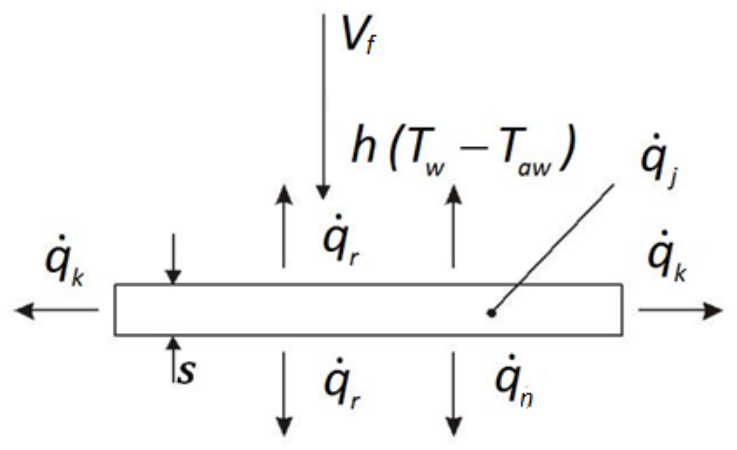

Fig. 4. Sketch of the local energy balance

The surface temperature distribution is measured by viewing the rear face of the foil (i.e. the opposite side of jet impingement). In fact, since the Biot number $B i=h s / \lambda_{f}$ (where $s$ and $\lambda_{f}$ are thickness and thermal conductivity of the foil, respectively) is small respect to unity, the temperature can be considered practically uniform across the foil thickness. Each test run consists of two parts: firstly, electric current off, $T_{\text {aw }}$ is measured and the so-called "cold image" is recorded; secondly, electric current on, $T_{w}$ is measured and the "hot image" is recorded. In particular, each image is averaged over 300 
measured fields and the quantity $T_{w}-T_{a w}$ is obtained by subtracting two thermal images, the cold one from the hot one. Besides, because of the thermal inertia of the foil and of the image time averaging process, present measurements have to be considered as time averaged, i.e. fluctuations due to turbulence are not being measured. The experimental data are reduced in dimensionless form in terms of Nusselt number $N u=h D / k$ ( $k$ is the thermal conductivity of air). Data are also presented as surface averaged Nusselt number $N u_{\text {mean }}$ and surface standard deviation percentage of Nusselt number $\sigma_{N u}$.

In particular $N u_{\text {mean }}$ and $\sigma_{N u}$ are evaluated as in eq. 2 and 3 on different circular areas (changing radius) with a fixed center identified as intersection of jet axis and target plate.

$$
\begin{gathered}
N u_{\text {mean }}(r)=\frac{1}{A_{r}} \int_{A_{r}} N u d A_{r} \\
\sigma_{N u}(r)=\frac{\sqrt{\int_{A_{r}}\left(N u-N u_{\text {mean }}\right)^{2} \cdot d A_{r}}}{\frac{1}{A_{r}} \int_{A_{r}} N u \cdot d A_{r}} \cdot 100
\end{gathered}
$$

$N u$ maps and radial distributions of $N u_{\text {mean }}$ and $\sigma_{N u}$ are used to quantify the convective heat transfer rate and the convective heat transfer uniformity and to deduce the flow field structure.

\section{Results}

In Fig. 5 are reported Nusselt number maps obtained for the cases of $S=0, S=0.4, S=0.6$ and $S=0.8$ for the distances $z=2, z=6$ and $z=10$. For sake of brevity only these maps are reported because they allow to well summarize all the most relevant aspects. In all the maps the center of impingement (intersection of jet axis and target plate) is used for surface averaging.

In the case of the multi-channel jet $(S=0), 2 D$ Nusselt number distributions show that the flow leaving the nozzle is divided into four separate jets due to the presence of the four channels within the insert; because of this, four distinct regions of stagnation can be seen on the plate, in the case $z=2$. For longer distances, however, the jets tend to merge and a single impingement area is observed. The multi-channel impinging jet provides very high heat transfer values.

For a Swirl number equal to 0.2 and 0.4 (weak swirl jets) similar behavior are observed (so only the maps for $S=$ 0.4 are reported). At the shortest considered distance, the effect of the swirl is not evident. Swirl appears to just produce a little decrease of heat transfer but the heat transfer topology still shows four high heat transfer separate regions with a low heat transfer uniformity; the distribution of Nusselt number doesn't appear being very different from the multichannel jet. With the increase of the nozzle to plate distance, it seems that the four jets merge at a short distance from the nozzle exit and seems to produce a more uniform heat transfer rate in stagnation region. The swirl flow seems to induce a broadening of the impingement region and of the wall jet region. In all cases the heat transfer over the whole observed area appears lower than the heat transfer obtained with the multi-channel jet; this is clearly related to the axial flux weakening caused by the swirl.

The jet with $S=0.6$ appears being in a condition of transition between the weak and the strong swirl. At all the considered distances the swirl is enough strong to produce a great broadening of the stagnation region, coupled with a decrease of heat transfer respect to swirling jets at lower swirl number. The impingement regions related to the four channels merge but not completely. In fact in the $N u$ map at $S=0.6$ and $z=6$ two high heat transfer lobes are still evident while the other two are small and seem to be close to merge with the bigger two. In the Nu map related to the case with $S=0.6$ and $z$ $=10$ only two lobes are present: they are highly decentralized and broadened (perhaps due to the merging). Even if the two lobes are still present the map shows a large region where the heat transfer is almost constant ( $N u$ ranging between 45 and 50).

In strong swirl phenomena, swirling flow produces axial pressure gradients that are more and more strong with the increase of swirl number and that can cause vortex breakdown [10] and the formation of a recirculation zone in the center of the jet as explained by Gupta [7] and shown by Alekseenko et al. measurements [11]. The maps reported in Fig. 5 related to $S=0.8$ well confirm these aspects; in fact in them, the distinction between the high heat transfer lobes is clear for all the values of the nozzle to plate distances analyzed. With the increase of $z$, the stagnation points gradually move away from the center of the plate producing a low heat transfer zone in the center of the plate. 

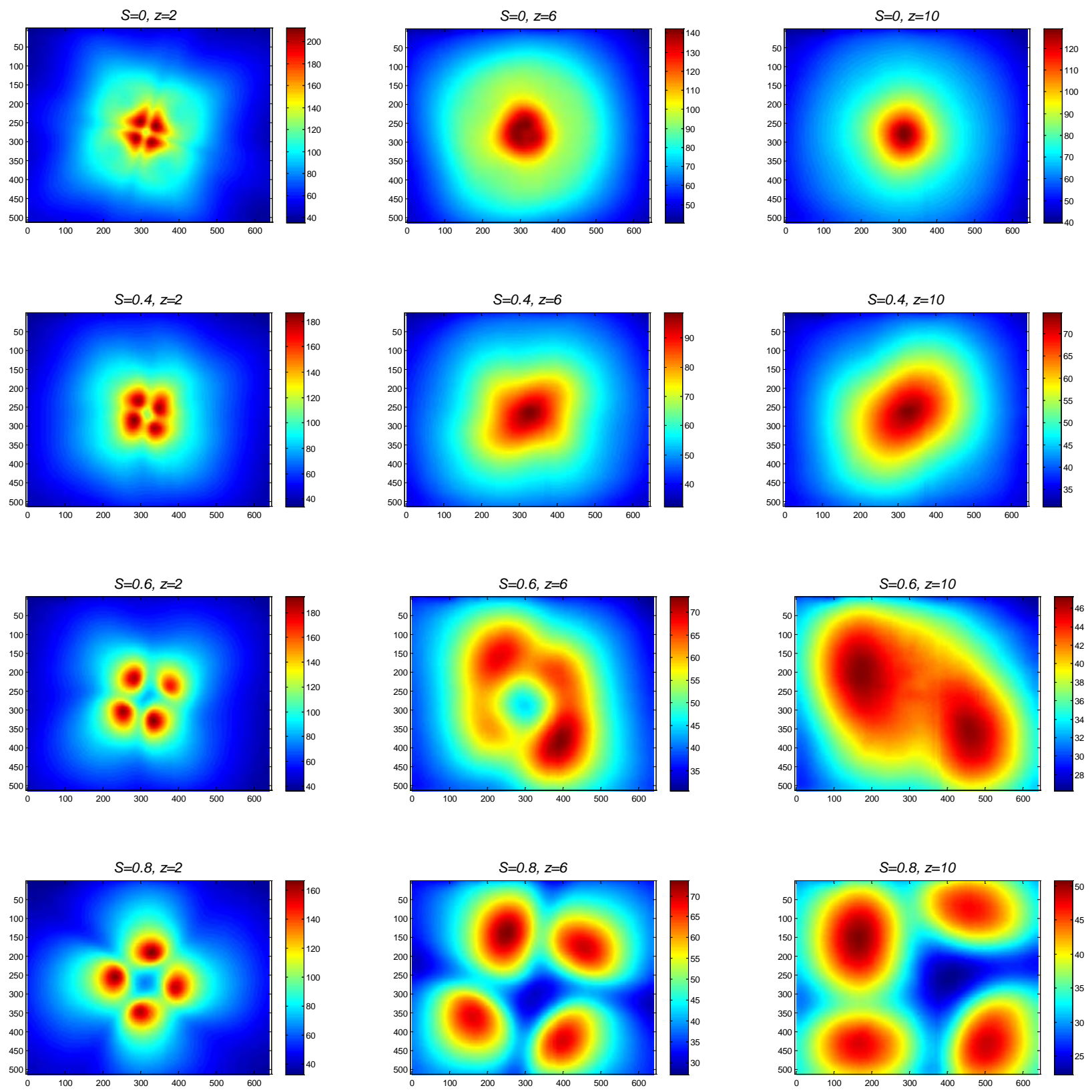

Fig. 5. Nu maps for $S=0, S=0.4, S=0.6$ and $S=0.8$ and $z=2, z=6$ and $z=10$

The influence of the swirl number and of the nozzle to plate distance on the heat transfer can be better analyzed by comparing the radial distribution of surface averaged Nusselt number and surface standard deviation percentage of Nusselt number. In Fig. $6 N u_{\text {mean }}(r)$ and $\sigma_{N u}(r)$ measured for all the five swirling nozzles are plotted for $z=2, z=6$ and $z=10$.

In the case of $z=2$ the swirl seems to induce just a displacement of the peak of heat transfer and a little decrease of global heat transfer. Moreover, the swirler do not change heat transfer non-uniformity. In fact if a zone on the impinged plate with radius equal to 3.5 nozzle diameters is considered, the surface averaged Nusselt number exhibits a difference between the cases $S=0$ and $S=0.8$ lower than $20 \%$ and the surface standard deviation percentage of Nusselt number obtained with swirl number equal to 0 is the lowest. 
With the increase of the nozzle to plate distance the effect of the swirl becomes stronger and more interesting and useful. In the case of $z=6$ the swirler with $S=0.6$ produces a $\sigma_{N u}$ absolutely low respect to the multi-channel jet but paying the price of a strong reduction of global heat transfer (about fifty percent).

At $z=10$ swirling nozzles, except the jet with $S=0.8$, show the best performances in heat transfer uniformity. The increase of $S$ always produce an increase of uniformity but is coupled with a decrease of global heat transfer.

At the highest swirl number the presence of the distinct zones of stagnation highly decentralized cause the highest standard deviation percentage. The best performances in term of low $\sigma_{N u}$ are obtained at swirl number equal to 0.4 and 0.6. In particular the $S=0.6$ jet shows an heat transfer almost constant over a zone on the impinged plate up to $r / D$ equal to 3.5 but with a reduction of about $50 \%$ of the global heat transfer respect to the multi-channel jet.
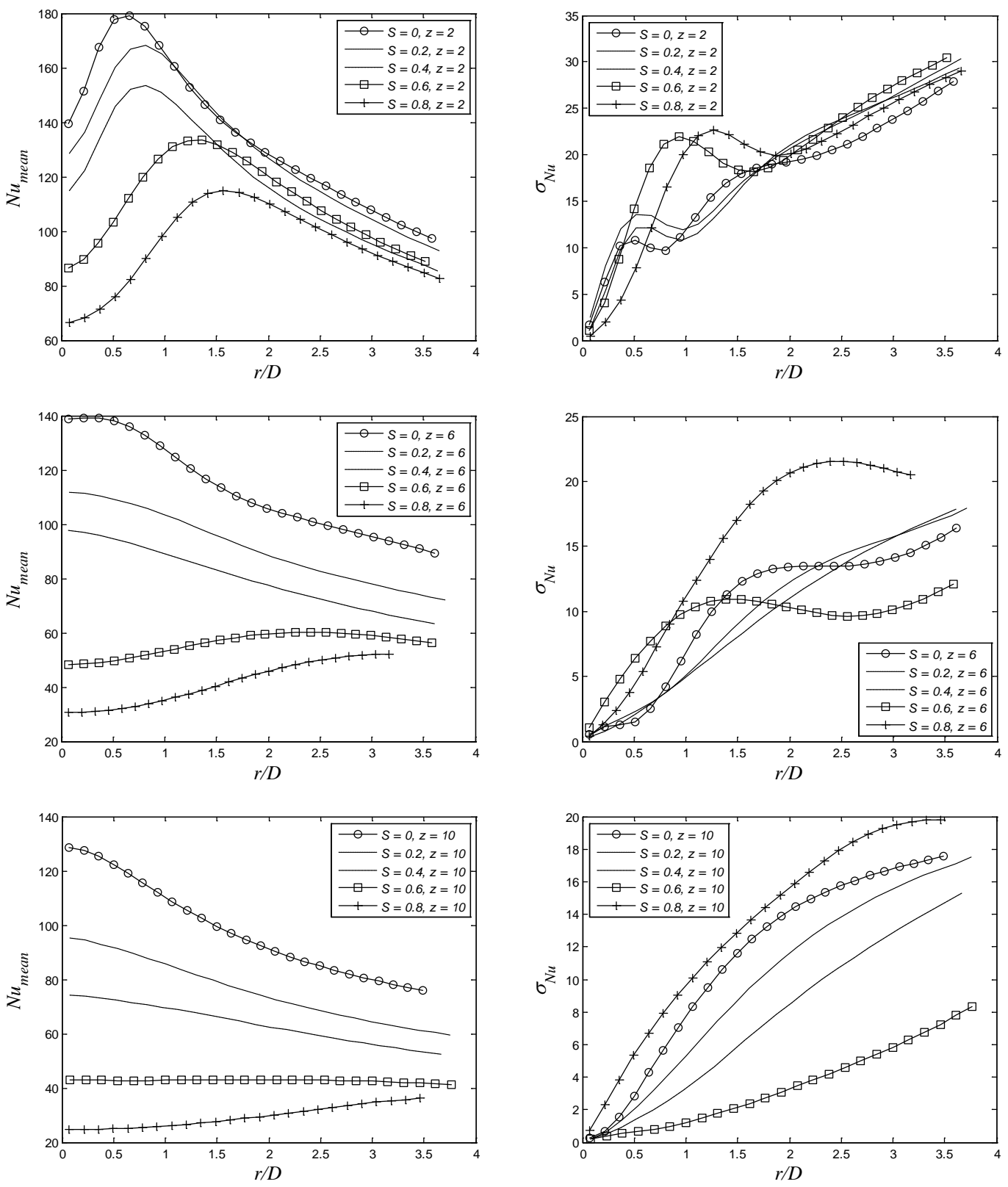

Fig. 6. $N u_{\text {mean }}$ and $\sigma(\mathrm{Nu})$ radial distributions at $z=2, z=6$ and $z=10$ 


\section{Conclusions}

In this work heat transfer measurements have been performed on a flat plate with swirling air jets impinging on it in order to analyze heat transfer rate and uniformity provided by the swirling jets. Through analysis of the standard deviation of $\mathrm{Nu}$, it was possible to quantitatively assess the uniformity of heat transfer for every case analyzed

In all the tested cases the mean heat transfer decreases with the increase of the nozzle to plate distance. With the increase of the distance there is an increase of the uniformity of the heat transfer.

Swirling jets are characterized by a broadening of the impingement region and, except the jet with $S=0.8$, at high $z$ show the best performances in heat transfer uniformity. The increase of $S$ produces an increase of uniformity but is coupled with a decrease of global heat transfer. Ultimately, studies highlight the peculiarity of the swirler with $S=0.6$ that shows an heat transfer almost constant.

\section{NOMENCLATURE}

\begin{tabular}{|c|c|c|}
\hline $\begin{array}{l}\text { Symbol } \\
\dot{m}\end{array}$ & $\begin{array}{l}\text { Quantity } \\
\text { Mass flow rate }\end{array}$ & $\begin{array}{l}\text { SI Unit } \\
K g / s\end{array}$ \\
\hline $\mathrm{Bi}$ & Biot numberBi=hs $/ \lambda_{f}$ & Dimensionless \\
\hline$D$ & Cylinder diameter & $m$ \\
\hline$h$ & Convective heat transfer coeff. & $W /\left(K m^{2}\right)$ \\
\hline$k$ & Air thermal conductivity & $W /(m K)$ \\
\hline $\mathrm{Nu}$ & Nusselt number $N u=h D / k$ & Dimensionless \\
\hline$N u_{\text {mean }}$ & Average $\mathrm{Nu}$ over a target area & Dimensionless \\
\hline $\operatorname{Re}$ & Reynolds number: $R e=4 \dot{m} /(\pi \mu D)$ & Dimensionless \\
\hline$S$ & Swirl number & Dimensionless \\
\hline$z$ & Nozzle to plate distance scaled by diameter $z=Z / D$ & Dimensionless \\
\hline$Z$ & Nozzle to plate distance & $m$ \\
\hline$\mu$ & Air viscosity & $K g /(m s)$ \\
\hline$\sigma_{N u}$ & Standard deviation percentage of $\mathrm{Nu}$ over a target area & Dimensionless \\
\hline
\end{tabular}

\section{REFERENCES}

[1] H. Martin, Heat and Mass Transfer between Impinging Gas Jets and Solid Surfaces, Advances in Heat Transfer, vol. 13, 1-60, 1977.

[2] C. Meola, A New Correlation of Nusselt Number for Impinging Jets, Heat Transfer Engineering, 30:3, $221-228$, 2009.

[3] R. Viskanta, Heat Transfer to Impinging Isothermal Gas and Flames Jets, Experimental Thermal and Fluid Science, vol. 6, 111-134, 1993.

[4] J. Ward, M. Mahmood, Heat transfer from a turbulent, swirling impinging jet, Proceedings of the 7th International Heat Transfer Conference, Vol. 3, 401-407, 1982.

[5] L. Huang and M. S. El-Genk, Heat transfer and flow visualization experiments of swirling, multi-channel, and conventional impinging jets, Int. J. Heat Mass Transfer, Vol. 41, No. 3, 583-600, 1998.

[6] D. H. Lee, S. J. Won, Y. T. Kim and Y. S. Chung, Turbulent Heat Transfer from a flat surface to a swirling round impinging jet, Int. J. Heat Mass Transfer, 45, 223-227, 2002.

[7] A. K. Gupta, D.G. Lilley and N. Syred, Swirl Flows, Abacus Press, 1984.

[8] M. Y. Wen and K. J. Jang, An impingement cooling on a flat surface by using circular jet with longitudinal swirling strips, International Journal of Heat and Mass Transfer,46, 4657-4667, 2003.

[9] G. M. Carlomagno and L. de Luca, Infrared Thermography in Heat Transfer, in Handbook of Flow Visualization, W.J. Yang ed., Hemisphere, New York, 531-553, 1989.

[10] P. Brillant, J. M. Chomaz and P. Huerre, Experimental study of vortex breackdown in swirling jets, J. Fluid Mech., 376, 183-219, 1998.

[11] S. V. Alekseenko, A.V. Bilsky, V. M. Dulin D. M. Markovich, Experimental study of and impinging jets with different swirl rates, International Journal of Heat and Fluid Flow, 28, 1340-1359,2007. 